L'intérêt de notre confrère est donc d'entrer dans le fond du débat et d'apporter des preuves sérieuses de l'hybridité de ses échantillons. Historiquement il ne saurait y avoir chose jugée : combien de fois l'exacte interprétation des faits de la nature n'a-t-elle pas été fort lente à se faire jour!

En ce qui concerne le nom de $P$. Saportæ, j’ai fourni les motifs le rendant obligatoirement modifiable en P. Saportæ-Marioni, si l'hybridité réelle du $P$. Lentisco-Terebinthus vient à n'ètre plus le problème que je désirerais voir M. G. Rouy solutionner au plus tôt.

M. Rouy dit qu'il n'a rien à ajouter à l'observation d'ordre purement historique qu'il a présentée à la séance du 10 mars, la question ayant été traitée par les botanistes qu'il a cités et de façon largement suffisante.

Lecture est donnée de la communication suivante :

\title{
De l'influence de la composition chimique du milieu sur la végétation de quelques Algues Chlorophycées,
}

PAR M. JOSEPH COMĖRE.

J'ai publié, en janvier 1903, le résultat de quelques recherches faites en vue d'étudier l'action des eaux salées sur les Algues d'eau douce et de démontrer que, moyennant certaines précautions, il était possible de faire vivre les Chlorophycées filamenteuses dans de l'eau de mer artificielle à divers degrés de salure ${ }^{1 .}$

Après ces premiers essais, tout en perfectionnant ma méthode de culture, j'en ai étendu l'application en employant des sclutions de matières salines diverses et examiné leur limite de toxicité, ainsi que les modifications qui pouvaient être déterminées dans l'état physiologique des espèces en expérience par les changements des conditions d'existence.

L'étude des rapports, entre la composition chimique du milieu

1. J. Comére. De l'action des eaux salées sur la végétation de quelques Algues d'eau douce (Nuova Notarisia, série 14, janvier 1903). 
et la végétation des Algues d'eau douce, a fait l'objet de plusieurs travaux et parmi les principaux publiés sur ce sujet spécial, sans mentionner ceux qui traitent exclusivement des composés organiques, je citerai ceux de M. Famintzine ${ }^{1}$ sur le développement des Algues dans des solutions de sels inorganiques; celles de M. Ritcher ${ }^{2}$ sur l'action spéciale du chlorure de sodium; de M. Wyples ${ }^{3}$ sur l'influence comparative des chlorures, bromures et fluorures et de M. Bonge ${ }^{4}$ sur celle du chlorure de sodium et des sels de fer.

MM. R. Boulllac ${ }^{3}$ et Molisch ${ }^{6}$ se sont occupés d'étudier le ròle joué par les arséniates dans l'alimentation des Algues unicellulaires; М. Воковму ${ }^{7}$ a examiné, entre autres substances, l'action des fluorure et fluosilicate de sodium et du bichlorure de mercure; N. YASUDA ${ }^{8}$, celle des chlorures de potassium, sodium et ammonium, sur l'Euglena viridis, et M. ONo ${ }^{9}$, celle de divers sels, en particulier du sulfate de fer, du sublimé corrosif, de quelques sulfates, fluorures, etc., etc.

MM. Matruchot et Molliard ${ }^{10}$ ont fait agir sur le Stichococcus

1. A. Fanintzine. Die anorganischen Salze, etc. (Bull. Acad.imp. sciences de Saint-Pétersbourg, VIII, 18\%1, pp. 226 et suiv, av., 3 pl.).

2. A. Ritcher. Ueber die Anpassung der Süsswasseralgen an Kochsalzlösungen. Inaugural Dissertation. München, 1892, 2 t.

3. M. Wrples. Ueber den Einfluss einiger Chloride, Fluoride und Bromide auf Algen. Waidhofen, 1893.

4. 0. Borge. Ueber die Rhizoidenbildung bei einigen fadenförmigen Chlorophyceen. Upsala, 1894.

כ. R. Bovillac. Influence de l'acide arsénique sur la végétation des Algues (Compt. rend. Acad. sciences, 26 nov. 1894, et Ann. agronomiques, XXIV, p. 561).

6. H. Molisch. Die Ernährung der Algen (Süswasseralgen, II, AbhandlungSitzungsber Kais. Akad. der Wissensch. in Wien. Mathem. naturw. Classe, Band CV, Abth. I, oct. 1896, p. 663-648).

7. Тн. BокоnNy. Vergleichende Studien über die Gittwirkung verschiedener chemischer Substanzen bei Algen und Infusorien (Archiv. für die Ges. Physiologie, Band 64, Bonn, 1896).

8. A. YASUDA. Studien ueber die Anpassungsfähigkeit einiger Infusorien an concentrirte Losüngen (Journ. Coll. Sc. Imp. Univ. Tokyo, vol. XIII, 1900, part. I, p. 101-140, pl. X-XII).

9. N. ONo. Ueber die wachsthumsbeschleunigung einiger Algen und Pilze durch chemische Reize (Journ. Coll. Sc. Imp. Univ. Tokyo, vol. XIII, 1900 , part. I, p. 141-186, pl. XIII).

10. L. Matruchot et M. Molliard. Variations de structure d'une Algue verte sous l'influence du milieu nutritif (Revue générale de botanique, XIV, 1902 , p. 193 et suiv.). 
bacillaris un certain nombre de matières organiques et des sels minéraux : les azotates de potassium et de calcium, le phosphate acide de potasse et le sulfate de cuivre.

Par mes recherches, en opérant sur des Algues filamenteuses d'une organisation relativement élevée et les cultivant dans des solutions nutritives additionnées de divers composés chimiques, j'ai essayé de démontrer qu'en graduant convenablement l'action des substances actives, on pouvait faire supporter aux espèces en expérience des doses assez élevées de celles-ci. Tout en déterminant l'action physiologique de chacun des sels employés, il m'a été permis de constater que certains d'entre eux pouvaient ètre tolérés dans une certaine mesure en acclimatant progressivement les végétaux cryptogamiques aux variations de composition chimique du milieu et que mème les solutions salines introduites dans mes cultures exerçaient à une dose déterminée une influence adjuvante sur la végétation des plantes.

Des méthodes perfectionnées de culture ont été appliquées aux Algues vertes protococcoïdes ${ }^{1}$. Ces organismes de forme très variable, offrant une succession de stades évolutifs, se laissent facilement cultiver et s'adaptent à des solutions salines très concentrées. En employant des précautions très minutieuses et les procédés en usage dans les études bactériologiques, on a pu suivre leur développement dans des conditions de pureté toutes spéciales.

Les formes supérieures en organisation, bien que plus faciles à reconnaître et à caractériser, sont d'une sensibilité beaucoup plus grande relativement et je me suis surtout

1. M. W. BEYERINCK. Kulturversuchen mit Zoochlorellen, Lichengonidien und anderen niederen Algen (Bot. Zeitung, nº 45, 1890). -. Beritcht über meine Kulturen niederen Algen auf Nährgelatine Centr. f. Bakt. und l'aras., t. XIII, 1893). - W. Krüger. Ueber zwei aus Saftflüssen rein gezüchtete Algen (Zopf's Beitrage z. Phys. u. Morph. nied.org., t.IV, p. 91). R. Chodit et J. Grintzesco. Sur les méthodes de culture des Algues vertes

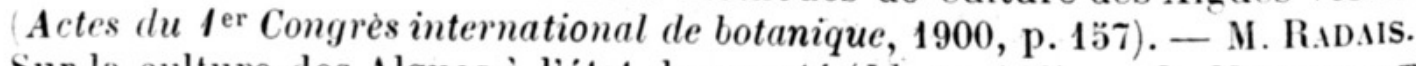
Sur la culture des Algues à l'état de pureté (Id., p. 163). - L. Matrcchot et M. Molliard, loco citat. 
préoccupé, tout en opérant avec le plus de soins possible, de les faire évoluer dans des conditions satisfaisantes en vue de comparer leur faculté variable d'adaptation.

Les solutions de Detmer, de Knopp, de MM. Chodat et GristzESCo, etc., peuvent être employées comme milieu de culture pour les Algues filamenteuses, mais il importe de les ramener à un degré de dilution convenable, car la proportion de sels solubles que ces liqueurs nutritives renferment est beaucoup trop élevée pour permettre un développement normal.

Les milieux naturels : l'eau des étangs et des marais, ou bien encore une macération de terre végétale, à l'exemple de M. Gerassinow ${ }^{1}$, sont aussi d'un emploi avantageux, mais les substances alimentaires qu'ils renferment, rapidement épuisées, ont bientôt fait défaut pour la nourriture des plantes et il faut renouveler le liquide nutritif très fréquemment.

J'emploie, de préférence à l'eau distillée qu'il n'est pas facile de se procurer pure et surtout exempte de sels de cuivre ${ }^{2}$, de l'eau de rivière stérilisée. L'eau de la Garonne, dont j'ai fait usage, ne renferme pas une proportion de sels très élevée. Le résidu salin est de $0 \mathrm{gr} .1367$ par litre, d'après les analyses de H. Deville ${ }^{3}$.

Mes flacons de culture, lavés au préalable à l'acide sulfurique et rincés avec soin, sont d'une capacité de 125 à 150 cc., bouchés à l'aide d'un tampon de coton, chauffés à l'étuve à $+110^{\circ}$ et reçoivent 100 cc. d'eau stérilisée, plus 30 gouttes d'une solution nutritive concentrée de la composition suivante :

Eau. . . . . . . . . . . 100

Azotate de calcium. . . . . . . . . . 0,25

Phosphate de potassium . . . . . . . . 0,23

Azotate de potassium. . . . . . . . . . 0,25

Sulfate de magnésium . . . . . . . . . . 0,25

Sulfate de fer. . . . . . . . Traces.

Carbonate de calcium, $Q$. S. pour neutraliser.

Cette solution doit ètre stérilisée au bain-marie à la tempéra-

1. J.-J. Gerassinow. Die Abhangigkett der Grosse der Zelle von der Menge ihrer Kernmasse Zeitschr. für allgem. Physiologie, Band I, 1902).

2. P.-P. Dehérain et E. Demoussy. La germination dans l'eau distillée (La Nature, 8 juin 1901, p. 19).

3. H. Deville. Ann. de Chimie et de Phys., t. XXIII, p. 32. 
ture de $70^{\circ}$ environ. Son degré de concentration la rend maniable et d'un dosage facile. Tous les cinq ou six jours, j'ajoute 3 à 弓̆ gouttes de liqueur nutritive aux cultures pour maintenir les bonnes conditions de vitalité et ne donner ainsi que la proportion de matières nécessaires au développement normal suivant les besoins de la plante.

L'aération fréquente des solutions de culture est aussi absolument nécessaire, car les aliments gazeux sont utiles à la synthèse organique. Il suffit d'employer dans ce but un dispositif permettant de faire passer, au travers du liquide de culture, un courant d'air formé par une trompe de laboratoire, ou tout autre moyen, et filtré au travers d'une couche de coton.

Avant de faire agir les substances actives, il est également bon de laisser les Algues s'acclimater pendant quelques jours au milieu nutritif. Ces essais d'adaptation donnent surtout de bons résultats avec les plantes 'en pleine végétation, et celles qui se préparent à la conjugaison ou à la sporulation tolèrent mal l'action des substances salines.

Les composés chimiques dont j'ai fait choix pour étudier leur action sur quelques Chlorophycées d'eau douce sont les suivants :

Chlorure de sodium.

Arséniate de sodium.

Iodure de potassium.
Bromure de potassium.

Sulfate de protoxyde de fer.

Les solutions de ces substances salines ont été dosées de manière à permettre l'introduction progressive dans les liquides de culture d'une quantité déterminée de matières actives. Au début des expériences, je n'ai fait agir qu'un petit nombre de gouttes des solutions titrées et, augmentant graduellement les proportions, je suis arrivé à employer plusieurs centimètres cubes à la fois. Ces additions doivent être convenablement espacées, car il est de beaucoup préférable de laisser un intervalle de temps convenable entre les introductions successives que de les faire quotidiennement à dose plus minime pour arriver au mème résultat. L'intervalle de temps entre les additions permet aux 
Algues de s'accoutumer graduellement aux changements amenés dans la concentration du milieu.

Les espèces suivantes :

Spiroyyra crassa Kütz.

S. Weberi Kütz.

OEdogonium capillare (Linn.) Kütz.

OEdogonium longatum Kütz.

Cladophora fracta kütz.

Conferva bombycina Ag.

ont été soumises à l'action des substances énumérées plus haut.

Depuis longtemps déjà, j'observe fréquemment ces plantes en vue de l'étude de leur évolution biologique. Les Spirogyra crassa et Weberi, l'OEdogonium capillare et le Cladophora fracta abondent à Toulouse dans le canal du Midi. Les bassins dans lesquels on cultive les plantes aquatiques au Jardin des plantes de notre ville me fournissent les mèmes Spirogyra crassa et Cladophora fracta et de plus le Conferva bombycina et l'OEdogonium longatum.

La structure délicate des chromoleucites du Spirogyra crassa rend cette espèce assez sensible aux variations de composition du milieu. L'OEdogonium capillare et le Spirogyra Weberi s'adaptent beaucoup mieux aux solutions salines. Il en est de même du Cladophora fracta et de l'OEdogonium longatum qui supportent l'action de solutions d'un degré de concentration encore plus élevé. Le maximum de résistance est fourni par le Conferva bombycina. Les facultés d'adaptation varient non seulement avec les divers groupes, mais aussi très sensiblement avec les espèces du même genre.

\section{III}

L'action sur les fonctions générales et sur les éléments de la cellule variant avec les diverses espèces en expérience et avec la nature des agents chimiques, il existe pour chaque plante une dose optimum et une dose nocive variables avec chacune des matières salines employées.

J'ai désigné par dose optimum la quantité de substance active que l'on ne peut dépasser sans arrèter le développement et ralentir les fonctions physiologiques, et par dose nocive la proportion de sels qui amène la désorganisation des éléments cellulaires et la mort. 
Sous l'influence de la dose optimum, la végétation des Algues filamenteuses est abondante, la coloration intense, l'accroissement continu et la production de nombreuses bulles d'oxygène, qui les maintiennent à la surface des liquides de culture, donnent une preuve de leur vitalité et de la persistance de leurs fonctions assimilatrices. Au delà de la dose optimum, l'accroissement est suspendu, la coloration se modifie et les filaments descendent peu à peu au fond des vases dans lesquels on les cultive.

Avant d'atteindre la dose nocive, et la dose optimum dépassée, les organismes préparent la résistance contre la concentration du liquide de culture, leur activité végétative est ralentie, ils accumulent des matières ternaires et leur membrane, subissant dans certains cas des déformations caractéristiques, présente des épaississements cellulosiques particuliers. Chez les $O E$ dogonium, le Cladophora et le Conferva, il se produit pendant cette période des formations de la même catégorie que les hypnocystes de F. G AY $^{1}$, akinètes de M. WILLE ${ }^{2}$ et qui comprennent les états de repos qui prennent naissance sans rajeunissement cellulaire et se montrent sous l'influence de conditions défavorables.

La dose nocive atteinte, la désorganisation totale s'opère rapidement.

Employées dans les limites de la dose optimum, aucune des solutions salines que j’ai expérimentées n'a exercé une action toxique sur les plantes soumises à leur influence. Au contraire, l'addition des sels solubles aux liquides de culture a produit, dans une proportion variable avec chaque substance et chaque espèce, une sorte d'accélération de la croissance que je crois pouvoir être interprétée comme le résultat d'une stimulation du tissu de l'enveloppe des Algues amenant une augmentation du pouvoir osmotique.

Il est à considérer aussi que les sels solubles, dont j'ai étudié l'action, entrent dans la composition des eaux de la mer et de certaines eaux minérales. Mais, sauf en ce qui regarde le chlo-

1. Fr. G.IY. Recherches sur le developpement de quelques Algues vertes, p. 11 et 12, Paris, 1891.

2. Wille. Ueber Akineten und Aplanosporen bei den Algen (Botan. Centralbl., XVI, p. 216, 1883). 
rure de sodium, ces composés salins ne se trouvent dans les milieux naturels qu'à des doses bien moindres que celles auxquelles j'ai adapté les Algues en expérience. Employés dans ces proportions, ils ne sont pas évidemment des aliments et il est possible d'admettre que leur addition aux milieux nutritifs produit une sorte d'influence adjuvante de la végétation, analogue aux phénomènes de chemauxisme constatés dans les cultures de divers organismes sous l'action de composés organiques et inorganiques.

En ce qui concerne en particulier l'arséniate de sodium, comme nous le verrons plus loin, son action nutritive, qui a été discutée en ce qui concerne les Algues protococcoïdes, m'a paru nulle pour les espèces filamenteuses supérieures.

Pour m'assurer de la bonne acclimatation des espèces soumises à l'action des divers agents chimiques, après avoir déterminé le degré de sensibilité de chaque forme pour chacun des sels employés, j'ai conservé les espèces adaptées aux doses optimum pendant un temps très long, en ayant soin d'ajouter au liquide de culture, à intervalles réguliers, quelques gouttes de la solution nutritive concentrée et à aérer de temps à autre le contenu des flacons. Les Algues se sont développées normalement et ont présenté tous les caractères d'une bonne végétation.

Ces mêmes Algues, transportées dans des milieux de composition égale à ceux auxquels elles étaient adaptées, ont continué à prospérer. Introduites dans des liquides amenés progressivement à un degré de concentration plus élevé, elles ont supporté l'action de doses de matières salines plus considérables que les plantes qui n'avaient pas été soumises à l'acclimatation préliminaire prolongée.

Les formes acclimatées sont au contraire rapidement détruites si on les ramène brusquement dans la solution nutrive normale, preuve certaine de leur parfaite adaptation à leurs nouvelles conditions d'existence.

\section{IV}

Les limites des doses optimum sont relativement faciles à déterminer, bien qu'elles soient en fonction des conditions de 
l'adaptation, mais celles des doses nocives sont plus difficiles à établir, surtout pour les formes aptes à développer des hypnocystes. Lorsque la production de ces formations spéciales a été provoquée dans de bonnes conditions par la concentration convenablement graduée des matières salines du milieu, les Algues peuvent ensuite supporter, sous cet état de vie latente, des proportions de sels très élevées et variables dans d'assez grandes limites. Il en résulte que les indications numériques que nous donnons, en nous occupant de l'action particulière de chaque substance, ne doivent pas ètre prises dans un sens absolu, mais n'ètre considérées que comme une évaluation surtout comparative des facultés de résistance des formes en expérience.

1. Action du chlorure de sodium. - A. Ritcher ${ }^{1}$ a établi que de nombreuses Algues d'eau douce appartenant aux groupes des Cyanophycées, des Diatomées et des Chlorophycées pouvaient se développer dans des solutions nutritives additionnées d'une quantité plus ou moins grande de chlorure de sodium. L'adaptation se fait d'autant mieux que l'organisation de l'Algue est moins compliquée. Le chlorure de sodium agit sur la coloration des chromoleucites. Certaines formes protococcoïdes, telles que : Chlorella, Tetraspora et Stichococcus, ont pu ainsi vivre dans des milieux contenant jusqu’à 8 p. 100 de sel marin.

L'action de plusieurs chlorures alcalins et alcalino-terreux, ainsi que de leur mélange, a été étudiée par $\mathrm{M}$. W $\mathrm{YPLEL}^{2}$. Comme A. Ritcher, M. W yplee a reconnu que les Algues supérieures offrent une résistance beaucoup moins grande à l'action des substances salines que les Algues inférieures.

Ainsi certains Spirogyra sont très sensibles, les OEdogonium et les Vaucheria beaucoup moins et les Cladophora moins encore. Les chlorures d'ammonium et de baryum exercent une action nuisible et, dans des solutions à 2 à 4 p. 100, les Protococcus meurent au bout de peu de temps. Les chlorures de calcium et de magnésium sont bien moins toxiques, et ceux de sodium, de potassium et de strontium sont encore bien mieux supportés.

D’après les expériences de $\mathbf{M}$. BorgE ${ }^{3}$, les Algues continuent

1. A. Ritcher, loco citat.

2. M. W YPLEL, loco citat.

3. O. BORGE, loco citat. 
à vivre dans une solution de chlorure de sodium, mais l'action de doses élevées entraîne leur mort.

M. Воковму ${ }^{1}$, étudiant l'action des fluorures alcalins, a vu que des Cladophora, OEdogonium et Oscillaria cessaient de vivre au bout de 24 heures dans une solution à 0,2 p. 100 de fluorure de sodium, tandis que ces mèmes Algues continuaient à prospérer dans une solution de chlorure de sodium de mème titre.

M. A. $\mathbf{Y}_{\mathrm{ASUdA}^{2}}{ }^{2}$ a constaté que l'Euglena viridis Ehr. présente une augmentation de volume et du nombre des chromatophores dans des solutions renfermant 0,8 p. 100 de chlorure de sodium, ou 0,7 p. 100 de chlorure de potassium, ou bien encore 0,6 p. 100 de chlorure d'ammonium. Les limites de résistance ont été de 1,8 p. 100 pour le chlorure de sodium, de 2,8 p. 100 pour le chlorure de potassium et de 1,4 p. 100 pour celui d'ammonium.

J'ai publié ${ }^{3}$ le résultat de quelques recherches que j'avais faites en vue d'étudier l'action des eaux salées sur quelques Chlorophycées. La solution employée pour introduire dans mes cultures une proportion déterminée de sels marins était celle dont le $\mathrm{D}^{\mathrm{r}}$ Miquel a fait usage dans ses recherches expérimentales sur la physiologie des Diatomées * Comme MM. A. Ritcher et 0 . Borge, j'avais constaté que le degré de résistance des Algues à l'action du chlorure de sodium est en raison inverse du degré de perfectionnement de la structure. Les Cladophora et les OEdogonium ont continué à vivre dans des milieux renfermant 2,כ̆ à $3, \breve{s}$ p. 100 de sels marins; les Vaucheria ont résisté à une concentration de 2 p. 100; les Spirogyra catenæformis et varians ont prospéré dans des solutions à 1,80 et 2 p. 100, mais les Spirogyra jugalis et orbicularis, formes à spires nombreuses et ténues, n'ont rapporté guère qu'une proportion de 1,כ0 p. 100.

Sous l'influence de la dose optimum, la coloration des chromoleucites passe au vert-jaune, cette teinte s'accentue avec la concentration de la dose de matière active.

Des renflements cellulaires anormaux et des déformations

1. Th. Bokorny, loco citat.

2. A. Yasuda, loco citat.

3. J. Così̀re, loco citat.

4. P. MiQuel. De la culture artificielle des Diatomées (Ann. de micrographie, t. IV, 1892). 
particulières se produisent fréquemment lorsque le degré de concentration des solutions de chlorure de sodium, atteignant une certaine limite, exerce une tension osmotique trop élevée. Ces modifications dans l'aspect général des plantes, qui ne contrarient nullement l'adaptation physiologique, se présentent, mais avec un degré moindre, sous l'action des autres sels solubles expérimentés.

Le chlorure de sodium est très facilement toléré par les Algues. Lorsque ce sel se trouve en proportion convenable, il intervient utilement pour favoriser la nutrition.

Tableau $\mathbf{I}$.

\begin{tabular}{|c|c|c|}
\hline \multirow{2}{*}{$\begin{array}{c}\text { ESPECES } \\
\text { EN EXPÉRIENCE }\end{array}$} & \multicolumn{2}{|c|}{ CHLORURE DE SODIUM } \\
\hline & $\begin{array}{cl}\text { Limites } \\
\text { de la dose optimum. }\end{array}$ & $\begin{array}{c}\text { Limites } \\
\text { de la dose nocive. }\end{array}$ \\
\hline Spirogyra crassa. . . . . . & $0, \ddot{30}$ à 0,60 p. 100 & 1,20 à 1,30 p. 100 \\
\hline Spirogyra Weberi. . & 1,73 à $2,00-$ & $3,7 \mathrm{a}$ à $4,75 \quad-$ \\
\hline OEdogonium capillare. & 1,25 à 1,50 - & 3,50 à 4,00 \\
\hline OEdogonium longatum. & 2,23 à $2,75 \quad-$ & 4,50 à $5,7 \mathrm{~b}-$ \\
\hline Cladophora fracta . . . . & 2,40 à $2,80-$ & 4,73 à 6,00 - \\
\hline Conferva bombycina. . . . & 2,30 à $3,00-$ & 5,50 à 6,50 - \\
\hline
\end{tabular}

2. Action de l'arséniate de sodium. - Après avoir constaté que les Phanérogames mouraient empoisonnées par l'acide arsénique, M. R. Bovillac ${ }^{1}$ a recherché si les arséniates étaient susceptibles de remplacer les phosphates dans la nutrition des Algues. Il a trouvé que, non seulement les arséniates ajoutés à une solution nutritive augmentaient la récolte, mais qu'en outre ces plantes se développaient très bien dans une solution privée de phosphates et renfermant des arséniates. Pour ces espèces végétales, les arséniates pourraient remplacer les phosphates.

Le mème auteur a reconnu que le Stichococcus bacillaris donnait en particulier des rendements plus élevés lorsque la solution nutritive renfermait 1 p. 1000 d'acide arsénique. Une dissolution contenant $0 \mathrm{gr}$. 001 d'acide phosphorique à l'état de phosphate de potasse ne donnait en moyenne que $0 \mathrm{gr} .40 \mathrm{de}$ Schizothrix lardacea pesé à l'ètat sec, tandis que l'on obtenait 
$1 \mathrm{gr} .80$ environ, si on ajoutait au liquide $0 \mathrm{gr} .50$ par litre d'arséniate de potassium.

D'après M. Molisch ${ }^{2}$, au contraire, le développement et la reproduction des Algues ne pourraient avoir lieu lorsque l'on remplace les phosphates par les arséniates. La quantité de ces derniers que les Algues peuvent tolérer est importante (plus de 2 p. 100), mais ceux-ci ne pourraient être substitués aux phosphates dans l'alimentation végétale.

J'ai essayé de cultiver l'OE'dogonium capillare dans une solution nutritive privée de phosphates et additionnée d'une certaine proportion d'arséniate alcalin. La plante a dépéri très rapidement et, dans un intervalle de temps très court, sa désorganisation a été complète. Au contraire, dans le milieu normal additionné d'arséniate de sodium, l'OEdogonium a bien végété, tant que la quantité ajoutée n'a pas dépassé les limites de la résistance à l'action du composé salin.

Pour être certain que, dans mes expériences, l'OEdogonium n'avait pas subi l'influence nocive d'une dose d'arséniate à laquelle il n'avait pas été adapté, j’ai placé l'Algue dans une solution privée de phosphates et je n'ai fait l'addition de l'arséniate que progressivement et dans les conditions qui ont servi de règle à tous mes essais. Les résultats ont été identiques et la plante n'a pu vivre dans le milieu qui ne renfermait pas de phosphates.

TABLEad II.



1. R. Boullate, loco citat.

2. M. Molisch, loco citat. 
3. Action de l'iodure de potassium. - Bokorny ${ }^{1}$ a reconnu que les corps halogènes étaient des toxiques très énergiques pour les Algues. Combinés aux alcalis, ils sont tolérés dans une certaine proportion par les espèces supérieures filamenteuses.

L'action de l'iodure de potassium s'est montrée analogue à celle du bromure et je n'ai pas observé de différence bien sensible dans la manière d'agir de ces deux composés.

La quantité d'iodure de potassium que peuvent supporter les Algues n'est pas très élevée, mais ce sel est beaucoup moins nocif comparativement, par exemple, que le sulfate de fer.

Sous l'influence de l'iodure de potassium, les chromoleucites prennent une teinte vert brillant caractéristique.

Tableav III.

\begin{tabular}{|c|c|c|}
\hline \multirow{2}{*}{$\begin{array}{c}\text { ESPÉCES } \\
\text { EN EXPÉRIENCE }\end{array}$} & \multicolumn{2}{|c|}{ IODURE DE POTASSIUM } \\
\hline & $\begin{array}{c}\text { Limites } \\
\text { de la dose optimum. }\end{array}$ & $\begin{array}{c}\text { Limites } \\
\text { de la dose nocive. }\end{array}$ \\
\hline Spirogyra crassa. . . . . . & 0,0 à 0,07 p. 100 & 0,15 à 0,20 p. 100 \\
\hline Spirogyra Weberi. . & 0,20 à $0,25 \quad-$ & 0,30 à 0,60 - \\
\hline OEdogonium capillare . . & 0,10 à $0,15-$ & 0,43 à 0,53 \\
\hline OEdogonium longatum. . . & 0,13 à 0,20 - & 0,60 à $0,75-$ \\
\hline Cladophora fracta... . & 0,25 à $0,30-$ & 0,60 à 0,83 \\
\hline Conferva bombycina.... & 0,20 à $0,30-$ & 0,75 à $0,90-$ \\
\hline
\end{tabular}

4. Action du bromure de potassium. - D'après les expériences de M. Wyples ${ }^{1}$, les bromures exercent une action toxique sur les Algues. Le plus actif serait le bromure d'ammonium, puis en ordre décroissant ceux de sodium et de potassium.

Au point de vue de la résistance physiologique et de la coloration des chromoleucites, les résultats que j'ai obtenus avec le bromure de potassium ne diffèrent pas sensiblement de ceux observés avec l'iodure de la mème base, bien que le premier de ces sels se soit montré moins actif.

1. M. WyPLEL, loco citat. 


\section{TABleaU IV.}

\begin{tabular}{|c|c|c|}
\hline \multirow{2}{*}{$\begin{array}{c}\text { ESPĖCES } \\
\text { EN EXPÉRIENCE }\end{array}$} & \multicolumn{2}{|c|}{ BROMURE DE POTASSIUM } \\
\hline & $\begin{array}{c}\text { Limites } \\
\text { de la dose optimum. }\end{array}$ & $\begin{array}{l}\text { Limites } \\
\text { de la dose nocive. }\end{array}$ \\
\hline Spirogyra crassa. & $0,0 \tau$ à 0,10 p. 100 & 0,20 à $0,2 \ddot{p}$ p. 100 \\
\hline Spirogyra Weberi. . & 0,25 à 0,30 - & 0,60 à $0,70-$ \\
\hline OEdogonium capillare . . & 0,12 à $0,20-$ & 0,50 à 0,73 — \\
\hline OEdogonium longatum. . & 0,20 à 0,23 & 0,75 à $0,90-$ \\
\hline Cladophora fracta . . . . & 0,30 à $0,40-$ & 0,70 à $0,8 \ddot{a}-$ \\
\hline Conferva bombycina. . . . & 0,25 à $0,30-$ & 0,75 à $1,20 \quad-$ \\
\hline
\end{tabular}

5. Action du sulfate de fer. - Les sels de fer exercent, d'après M. 0. Borge ${ }^{1}$, une action nuisible sur les Algues à une dose peu élevée et le sulfate ferreux, d'après М. Тн. Воковму ${ }^{2}$, aurait été toléré par des Spirogyra pendant plusieurs heures à la dose de $0 \mathrm{gr} .10 \mathrm{p} .100$.

Dans ses recherches opérées sur des Hormidium, M. N. Oxo a constaté que l'action optimum du sulfate ferreux avait lieu à la dose de 0,0005 p. 100, et la limite de résistance à celle de 0,0126 p. 100.

TABLEAU $\mathbf{V}$.

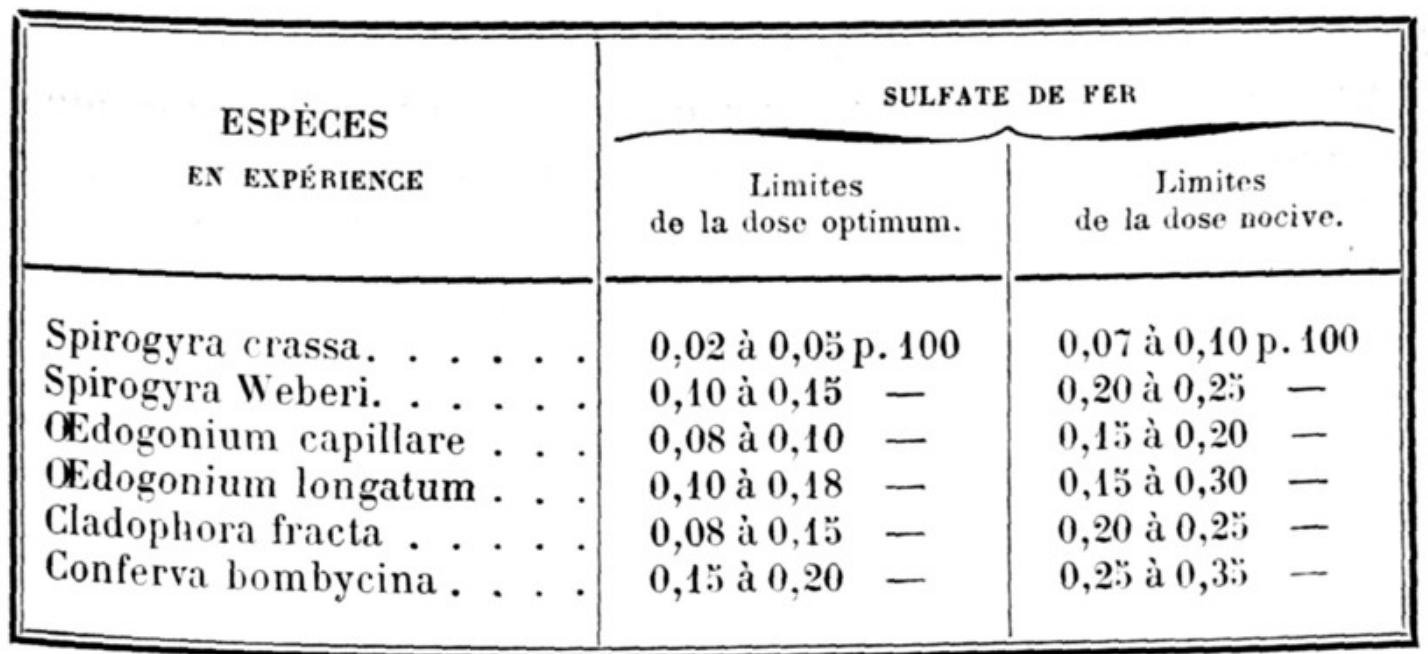

Le sulfate de fer est le plus actif des agents chimiques que j'ai expérimentés. A très faible dose, il exerce une action très

1. 0 . BORGE, loco citat.

2. Тн. BoкоRNY, loco citat. 
favorable sur la végétation des Algues et sur la coloration des chromoleucites, dont il avive considérablement la teinte verte; mais, pour réussir les essais d’adaptation, il faut employer des précautions particulières et, pour éviter l'altération de la solution de sulfate de fer, j'ai additionné celle-ci d'une très petite proportion d'acide citrique.

De plus, dans les observations d'une certaine durée, il est indispensable de renouveler assez fréquemment le contenu des flacons de culture. Un dépôt ferrugineux d'oxyde de fer peut former à la longue sur les filaments des plantes une sorte de gaine ocracée protectrice, qui leur permet de supporter des doses relativement élevées de sulfate ferreux et sensiblement supérieures à celles qui représentent les limites de la résistance physiologique dans les conditions normales.

Il me sera permis, d'après les expériences auxquelles j’ai procédé, de formuler les conclusions suivantes :

$1^{\circ}$ Le degré de résistance physiologique des Algues supérieures filamenteuses d'eau douce à l'action des solutions salines, tout en étant beaucoup moins élevé que celui des formes protococcoïdes, est variable avec les divers agents chimiques, les diverses espèces du mème genre et en fonction de la graduation progressive dans l'action des matières salines.

$2^{\circ}$ On peut déterminer pour chaque plante et pour chaque composé chimique les proportions quantitatives de substances actives correspondant aux limites des doses optimum et nocive.

La dose optimum comprenant la quantité de matière active qui peut être ajoutée progressivement à la solution nutritive sans que la végétation et les fonctions vitales de l'Algue en suspension dans le liquide soient modifiées ou ralenties. En opérant dans des conditions favorables d'adaptation et en ne dépassant pas les limites de la dose optimum, l'on peut faire vivre et prospérer les espèces en expérience dans les liquides de culture additionnés de diverses matières salines et réaliser une véritable acclimatation aux nouvelles conditions de milieu.

La dose nocive désigne la proportion des sels qui amène la désorganisation et la mort des éléments cellulaires. 
$3^{\circ}$ Il y a lieu d'ajouter une grande importance à l'emploi des précautions à prendre dans les essais d'adaptation, les résultats pouvant être rendus inexacts par une action trop rapide ou insuffisamment graduée et par les mauvaises conditions des milieux de culture.

$4^{\circ}$ Les sels, dont j’ai étudié l'action, employés dans une proportion ne dépassant pas les limites de la dose optimum, produisent une sorte d'influence adjuvante dans la végétation, une accélération de croissance des plantes en expérience et une action particulière sur la coloration des chromoleucites variable avec les diverses espèces.

Le chlorure de sodium est très favorablement toléré par les Algues filamenteuses d'eau douce.

L'arséniate de sodium, les bromure et iodure de potassium et le sulfate de fer sont en ordre croissant plus actifs, et leur action nocive s'exerce à des doses bien moindres.

$5^{\circ}$ En ce qui concerne plus particulièrement l'action des sels arsénicaux sur les Chlorophycées filamenteuses, j’ai constaté que ces composés ne pouvaient pas remplacer les phosphates dans les liquides nutritifs et que les plantes dépérissaient dans un milieu dans lequel on substituait à ces sels les arséniates alcalins.

$6^{\circ} \mathrm{Au}$ point de vue de la résistance physiologique des espèces observées, nous pouvons placer au premier rang le Conferva bombycina et le Cladophora fracta, ensuite, en ordre décroissant, l'OEdogonium longatum, le Spirogyra Weberi, l'OEdogonium capillare et le Spirogyra crassa, cette dernière forme étant très sensible à l'action des différents réactifs.

M. Lutz donne lecture de la communication suivante :

\section{Notes lichénologiques,}

\section{PAR LE Dr M. BOULY DE LESDAIX.}

Le signe (*) indique des espèces ou variétés nouvelles pour la France.

*Evernia divaricata (L.) Ach. subsp. E. Illyrica. A. Zahlb. Sched. ad Krypt. exsicc. edit. a Mus. Palat. Vindob., 1904, p. 418, et Krypt. exsicc. edit. a Mus. Palat. Vindob., no 1049 (Littore austriaco :

T. LII.

SÉANCES) 16 


\section{$2 \mathrm{BHL}$ Biodiversity Heritage Library}

Comère, Joseph. 1905. "De l'influence de la composition chimique du milieu sur la végétation de quelques Algues Chlorophycées." Bulletin de la Société botanique de France 52, 226-241.

https://doi.org/10.1080/00378941.1905.10829152.

View This Item Online: https://www.biodiversitylibrary.org/item/8674

DOI: https://doi.org/10.1080/00378941.1905.10829152

Permalink: https://www.biodiversitylibrary.org/partpdf/160510

\section{Holding Institution}

Missouri Botanical Garden, Peter H. Raven Library

\section{Sponsored by}

Missouri Botanical Garden

\section{Copyright \& Reuse}

Copyright Status: Public domain. The BHL considers that this work is no longer under copyright protection.

This document was created from content at the Biodiversity Heritage Library, the world's largest open access digital library for biodiversity literature and archives. Visit BHL at https://www.biodiversitylibrary.org. 